\title{
LE JEU DES (DIS)CONTINUITÉS ENTRE ARCHITECTURE ET SYSTÈMES SOCIAUX PEUT-IL NOUS SORTIR DE L'ANTHROPOCÈNE ?
}

\author{
Author(s) / Auteur(s) : \\ Damien CLAEYS \\ Architecte, Docteur en art de bâtir et urbanisme, Chargé de cours \\ Faculté d'architecture, d'ingénierie architecturale, d'urbanisme (UCL) \\ damien.claeys@uclouvain.be
}

Keywords / Mots-clés :

architecture, système, société, utopie, anthropocène

La relation générale entre les systèmes sociaux et l'environnement - au sens large - a favorisé l'émergence de l'ère de l'anthropocène. Pour un nombre croissant de scientifiques, l'émergence de la période la plus récente des temps géologiques - l'anthropocène - est clairement liée au développement des activités humaines qui contribuent à la destruction irréversible des ressources nécessaires à la survie de nombreuses espèces, dont l'espèce humaine. Cette hypothèse scientifique est confirmée par un nombre croissant de preuves indiquant que les processus atmosphériques, géologiques, hydrologiques et biosphériques du système terrestre sont aujourd'hui altérés par les humains.

La relation particulière entre les systèmes sociaux et l'environnement - cette fois construit - participe également à l'émergence de l'anthropocène. La somme des édifices construits (l'architecture), des chantiers d'envergure (l'ingénierie) et des villes (l'urbanisme) représente un poids non négligeable sur le système terrestre. La compréhension des relations entre systèmes sociaux et environnements construits est un enjeu contemporain important qui nécessite des méthodes pour penser et des outils pour agir innovants !

Une boucle de rétroaction est à l'oeuvre entre les systèmes sociaux et l'architecture : les systèmes sociaux produisent des établissements humains matérialisés par des artefacts architecturaux et, à leur tour, ces édifices structurent les membres de la société qui les habitent, ces derniers proposant alors de nouveaux types d'édifices et ainsi de suite... Ces influencent réciproques ne viennent pas d'actes isolés mais résultent d'un grand nombre d'actes conscients et inconscients s'enchaînant et interagissant. Ce fonctionnement complexe implique l'hypothèse de l'existence potentielle de (dis)continuités entre les structures sous-jacentes de cet environnement construit et des systèmes sociaux qu'il abrite, c'est à dire l'apparition potentielle de facteurs de cohérence communs aux systèmes sociaux et à leurs manifestations matérielles.

Sur base de cette hypothèse, s'ils connaissent les leviers de ces facteurs de cohérence communs, les concepteurs de l'environnement construit, décident implicitement de prolonger ou de transformer les propriétés des systèmes sociaux actuels, par les édifices qu'ils projettent et les réponses architecturales qu'ils proposent aux questions de société actuelles. Ils partagent donc indirectement la responsabilité de l'état d'épuisement du système terrestre qui affecte l'anthropocène.

Les systèmes sociaux sont en mutation constante : la population augmente et les acteurs sont multipliés, les ressources s'épuisent et la soutenabilité est nécessaire, les connaissances s'accumulent et les mémoires externes sont croisées, les idées circulent et la noosphère se développe, les règles se confondent, les clés de lecture et les codes culturels sont multiples, la ville intelligente se fragmente, la smartcity est diffuse, l'économie se dédouble et le capitalisme engendre l'ubérisation, la science se perd parce que les incertitudes s'accumulent à très grande et à très petite échelle. En Occident, la diffusion généralisée des technologies numériques amène les acteurs à perdre en partie leurs capacités mentales de mémorisation et de traitement de l'information. Par contre, ils gagnent la possibilité de 
vivre des mises en relation autrefois inédites et également une faculté décuplée d'invention et de création (Serres, 2013).

Parallèlement à ces évolutions, l'environnement construit est également en mutation constante, avec l'émergence de villes intelligentes, d'éco quartiers, de changements d'activités... Deux positions sont prise par enrayer le phénomène de l'anthropocène :

1. d'un côté, ceux qui s'opposent aux effets négatifs de la grande accélération entamée après la Seconde Guerre mondiale et qui proposent une slow architecture imprégnée de régionalisme critique, de low technologie et de ré-enchantement du monde,

2. de l'autre, ceux qui surfent sur les effets positifs de cette grande accélération et qui proposent une smart architecture encombrée de fonctionnalisme, de technologies, de capteurs et de régulations multiples. Malheureusement, les premiers réalisent des parenthèses (très) couteuses appréciables par des initiés dans des implantations uniques, alors que les seconds réalisent des interventions de grande ampleur (très) couteuses adressée uniquement à de jeunes cadres dynamiques et connectés.

Ces deux voies mènent à terme à une impasse. Une alternative est nécessaire !

Ces modifications touchent également le domaine de l'architecture, en tant que système particulier de mise en ordre de la réalité. L'accumulation et la complication des pratiques et des savoirs extériorisés, l'accroissement du nombre des acteurs et des facteurs pris en considération dans la conception, les contradictions liées à la construction d'une réalité contingente... sont autant d'influences de la société sur la discipline.

Pour l'architecture, l'un des défis majeurs de notre époque est d'appréhender et surtout de gérer la complexité inhérente à ces constats. À la tradition épistémologique occidentale héritée du cartésianisme correspond une conception réductrice du monde. Notre vision de la réalité doit être complétée de cadres de pensée fondés sur d'autres représentations qui visent sa complexité. Il est dorénavant possible d'éclairer le domaine de l'architecture par des concepts et des méthodologies qui permettent de bien penser dans l'action, avec lucidité et en pleine conscience de l'interdépendance des phénomènes.

À l'occasion du cinq-centième anniversaire de la parution de l'Utopie (1516) de Thomas More, le concept d'utopie est largement remis à l'honneur. Le concept d'utopie est terriblement stimulant pour les concepteurs de projets d'architecture puisqu'il désigne au départ un lieu inexistant tout en étant imaginable. En questionnant les modalités d'organisation spatiale de l'environnement construit, il confronte inévitablement l'architecture aux systèmes sociaux des cités qu'elle génère indirectement, mais qu'elle sert également. Dans le monde entier, les problèmes actuels que posent les villes (la pauvreté, la barbarie, la pollution...) démontrent l'actualité de la question de l'utopie.

Jusqu'à preuve du contraire, l'espèce humaine n'a actuellement pas encore disparu ! Espérons que l'usage de l'utopie critique combinée à la puissance holistique de la pensée systémique soit à l'oeuvre aujourd'hui !

\section{RÉFÉRENCES}

SERRES, M. (2013). "L'innovation et le numérique". Conférence inaugurale du Programme Paris Nouveaux Mondes, l'Initiative d'excellence du Pôle de recherche et d'enseignement supérieur "hautes études, Sorbonne, arts et métiers" (Pres héSam), le 29 janvier 2013. 